\title{
THREE- AND FOUR-STAGE IMPLICIT INTERVAL METHODS OF RUNGE-KUTTA TYPE
}

\author{
KAROL GAJDA ${ }^{1}$, ANDRZEJ MARCINIAK ${ }^{2}$, BARBARA SZYSZKA ${ }^{1}$ \\ ${ }^{1}$ Institute of Mathematics, ${ }^{2}$ Institute of Computing Science \\ Poznań University of Technology \\ Piotrowo 3a, 60-965 Poznań, Poland
}

\begin{abstract}
The paper presents three- and four-stage implicit interval methods of Runge-Kutta type and is a continuation of our previous paper [6] dealing with one- and two-stage methods of this kind. It is shown that the exact solution of the initial value problem belongs to interval-solutions obtained by both kinds of these methods (three- and four-stage). We also present some approximations of the widths of interval-solutions.
\end{abstract}

\section{INTRODUCTION}

Interval methods for solving the initial value problem are interesting due to interval-solutions obtained by such methods which contain their errors. Computer implementations of interval methods in floating-point interval arithmetic together with the representation of initial data in the form of minimal machine intervals, i.e. by intervals which ends are equal or neighboring machine numbers, yield interval solutions which contain all possible numerical errors.

In this paper we consider three- and four-stage implicit interval methods of Runge-Kutta type, which are presented in Sections 3 and 4. As we proved in [6], the exact solution of the initial value problem belongs to interval-solutions obtained by both kinds of these methods. In Sect. 5 some approximations of the widths of interval-solution are given.

\section{THE INITIAL VALUE PROBLEM AND CONVENTIONAL RUNGE-KUTTA METHODS}

As is well-known (see e.g. [5]), the initial value problem consists in finding the function $y-y(x)$, such that

$$
y^{\prime}=f(t, y(t)), \quad y(0)=y_{0},
$$

where $t \in[0, T], y \in \mathbf{R}^{N}$ and $f:[0, T] \times \mathbf{R}^{N} \rightarrow \mathbf{R}^{N}$. We will assume that the solution of(1) exists and is unique.

To carry out a single step by a conventional, $m$-stage Runge-Kutta method we apply the formula (see e.g. [1])

$$
y_{k+1}=y_{k}+h \sum_{i=1}^{m} w_{i} \kappa_{i}, \quad k=0,1, \ldots,
$$


where

$$
\begin{gathered}
\kappa_{i}=f\left(t_{k}+c_{i} h, y_{k}+h \sum_{j=1}^{s} a_{i j} \kappa_{j}\right), \quad i=1,2, \ldots, m, \\
\sum_{i=1}^{m} w_{i}=1, \quad c_{i}=\sum_{j=1}^{s} a_{i j}
\end{gathered}
$$

and where $s=i-1$ for an explicit method, and $s=m$ for an implicit one. The set of numbers $w_{i}, \mathrm{c}_{i}, a_{i j}$ are constants which characterize a particular method.

The local truncation error of step $k+1$ for a Runge-Kutta method (explicit and implicit) of order $p$ can be written in the form (see e.g. [1], [3] or [5])

$$
\begin{aligned}
r_{k+1}(h) & =\psi\left(t_{k}, y\left(t_{k}\right)\right) h^{p+1}+O\left(h^{p+2}\right) \\
& =r_{k+1}^{(p+1)}(0) \frac{h^{p+1}}{(p+1) !}+r_{k+1}^{(p+2)}(\theta h) \frac{h^{p+2}}{(p+2) !},
\end{aligned}
$$

where

$$
0<\theta<1,\left|\frac{r_{k+1}^{(p+2)}(\theta h)}{(p+2) !}\right| \leq M .
$$

This error is equal to the difference between the exact value $y\left(t_{k}+h\right)$ and its approximation evaluated on the basis of the exact value $y\left(t_{k}\right)$. The function $\psi(t, y(t))$ depends on coefficients $w_{i}, c_{i}, a_{i j}$, and on partial derivatives of the function $f(t, y)$ occuring in (1). The form of $\psi(t, y(t))$ is very complicated and cannot be written in a general form for an arbitrary $p$ (see e.g. [1], [5] or [8]).

\section{THREE-STAGE IMPLICIT INTERVAL METHODS}

Let us denote:

$\mathrm{A}$, and $\mathrm{A}$ - sets in which the function $f(t, y)$ is defined, i.e.

$$
\begin{gathered}
\Delta_{t}=\{t \in \mathrm{R}: 0 \leq t \leq a\}, \\
\Delta_{y}=\left\{y=\left(y_{1}, y_{2}, \ldots, y_{N}\right)^{T} \in \mathrm{R}^{N}: \underline{b}_{i} \leq y_{i} \leq \bar{b}_{i}, \quad i=1,2, \ldots, N\right\},
\end{gathered}
$$

$F(T, Y) \quad$ - an interval extension of $f(l, y)$,

$\psi(T, Y)$ - an interval extension of $\psi(t, y)$ (see (5)),

and let us assume that:

- the function $F(T, Y)$ is defined and continuous for all $T \subset \Delta_{\text {, }}$ and $Y \subset \Delta_{y}$,

- the function $F(T, Y)$ is monotonic with respect to inclusion, i.e.

$$
T_{1} \subset T_{2} \wedge Y_{1} \subset Y_{2} \Rightarrow F\left(T_{1}, Y_{1}\right) \subset F\left(T_{2}, Y_{2}\right)
$$


- for each $T \subset \Delta_{t}$ and for each $Y \subset \Delta_{y}$ there exists a constant $\mathrm{L}>0$ such that

$$
d(F(T, Y)) \leq L(d(T)+d(Y))
$$

where $d(A)$ denotes the width of $A$ (if $\left.A_{1}, A_{2}, \ldots, A_{N} \quad\right)^{T} \quad$, then the number $d(A)$ is defined by $\left.d(A)=\max _{i=1,2, \ldots, N} d\left(A_{i}\right)\right)$,

- the function $\Psi(T, Y)$ is defined for all $T \subset \Delta_{t}$ and $Y \subset \Delta_{y}$,

- the function $\Psi(T, Y)$ is monotonie with respect to inclusion.

From the theory of conventional Runge-Kutta methods it is known that two-stage implicit methods, which are characterized by the set of coefficients $w_{i}, c_{i}, \mathrm{a}_{i j}$, where $i, j=1,2,3$, can have order up to six, and the maximum order condition $(p=6)$ is achieved if (see e.g. [1] or [3])

$$
\begin{gathered}
w_{1}=w_{3}=\frac{5}{18}, \quad w_{2}=\frac{4}{9}, \quad c_{1}=\frac{1}{2}-\frac{\sqrt{15}}{10}, \quad c_{2}=\frac{1}{2}, \quad c_{3}=\frac{1}{2}+\frac{\sqrt{15}}{10}, \\
a_{11}=a_{33}=\frac{5}{36}, \quad a_{12}=\frac{2}{9}-\frac{\sqrt{15}}{15}, \quad a_{13}=\frac{5}{36}-\frac{\sqrt{15}}{30}, \\
a_{21}=\frac{5}{36}+\frac{\sqrt{15}}{24}, \quad a_{22}=\frac{2}{9}, \quad a_{23}=\frac{5}{36}-\frac{\sqrt{15}}{24}, \quad a_{31}=\frac{5}{36}+\frac{\sqrt{15}}{30}, \quad a_{32}=\frac{2}{9}+\frac{\sqrt{15}}{15} .
\end{gathered}
$$

In general, for $t_{0}=0$ and $y_{0} \in Y_{0}$ three-stage implicit interval method of Runge-Kutta type we define as follows:

$$
\begin{aligned}
Y_{n}\left(t_{0}\right) & =Y_{n}(0)=Y_{0}, \\
Y_{n}\left(t_{k+1}\right) & =Y_{n}\left(t_{k}\right)+h\left(w_{1} K_{1, k}(h)+w_{2} K_{2, k}(h)+w_{3} K_{1, k}(h)\right) \\
& +\left(\Psi\left(T_{k}, Y_{n}\left(t_{k}\right)\right)+[-\alpha, \alpha]\right) h^{p+1}, \\
k & =0,1, \ldots, n-1,
\end{aligned}
$$

where $p \leq 6$, and

$$
\begin{aligned}
K_{i, k}(h) & =F\left(T_{k}+c_{i} h, Y_{n}\left(t_{k}\right)+h\left(a_{i 1} K_{1, k}(h)+a_{i 2} K_{2, k}(h)+a_{i 3} K_{3, k}(h)\right)\right), \\
i & =1,2,3, \quad \alpha=M h_{0} .
\end{aligned}
$$

The step-size $h$ of the method (7), where $0<h \leq h_{0}, h_{0}$ denotes a given number, is calculated from the formula

$$
h=\frac{\eta_{3}^{*}}{n},
$$

where

$$
\eta_{3}^{*}=\min \left\{\eta_{0}, \eta_{1}, \eta_{2}, \eta_{3}\right\}
$$

The numbers $\eta_{i}>0(i=1,2,3)$ are evaluated in such a way that 


$$
Y_{0}+\eta_{i} c_{i} F\left(\Delta_{i}, \Delta_{y}\right) \subset \Delta_{y}, \quad i=1,2,3,
$$

and the number $\eta_{0}-$ from the relation

$$
\begin{gathered}
Y_{0}+\eta_{0}\left(w_{1} F\left(\Delta_{1}, \Delta_{y}\right)+w_{2} F\left(\Delta_{t}, \Delta_{y}\right)+w_{3} F\left(\Delta_{l}, \Delta_{y}\right)\right) \\
+\left(\Psi\left(\Delta_{l}, \Delta_{y}\right)+[-\alpha, \alpha]\right) h_{0}^{p} \subset \Delta_{y},
\end{gathered}
$$

for $Y_{0} \subset \Delta_{y}$ and $y_{0} \in Y_{0}$.

The interval $\left[0, \eta_{3}^{*}\right]$ wedivideinto $n$ parts by the points $t_{k}=k h(k=0,1, \ldots, n)$, and the intervals $\boldsymbol{T}_{\boldsymbol{k}}$, which appear in the method (7) - (8), we choose in such a way that

$$
t_{k}=k h \in T_{k} \subset\left[0, \eta_{3}^{*}\right] \text {. }
$$

From (8) it follows that in each step of the method we have to solve a (vector) interval equation of the form

$$
\mathrm{X}=\mathrm{G}(\mathrm{T}, \mathrm{X})
$$

where

$$
\begin{gathered}
T \in \Delta_{t} \subset I(\mathbf{R}), X=\left(X_{1}, X_{2}, \ldots, X_{N}\right)^{T} \in I\left(\Delta_{y}\right) \subset I\left(\mathbf{R}^{N}\right) \\
G: I\left(\Delta_{1}\right) \times I\left(\Delta_{y}\right) \rightarrow I\left(\mathbf{R}^{N}\right) .
\end{gathered}
$$

If we assume that the function $\mathrm{G}$ is a contraction mapping, then the corresponding iteration process follows from the fixed-point theorem.

\section{FOUR-STAGE IMPLICIT INTERVAL METHODS}

Four-stage implicit methods, which are characterized by the set of coefficients $w_{i}, c_{i}, \mathrm{a}_{i j}$, $(i, j-1,2,3,4)$, can have order up to eight, and if (see e.g. [3])

$$
\begin{gathered}
w_{1}=w_{4}=\frac{1}{4}-\frac{\sqrt{30}}{72}, \quad w_{2}=w_{3}=\frac{1}{4}+\frac{\sqrt{30}}{72}, \\
c_{1}=\frac{1}{2}-A_{1}, \quad c_{2}=\frac{1}{2}-B_{1}, \quad c_{3}=\frac{1}{2}+B_{1}, \quad c_{4}=\frac{1}{2}+A_{1}, \\
a_{11}=a_{44}=\frac{w_{1}}{2}, \quad a_{12}=\frac{w_{2}}{2}-A_{2}+B_{3}, \quad a_{13}=\frac{w_{2}}{2}-A_{2}-B_{3}, \quad a_{14}=\frac{w_{1}}{2}-A_{4}, \\
a_{21}=\frac{w_{1}}{2}-B_{2}+A_{3}, \quad a_{22}=a_{33}=\frac{w_{2}}{2}, \quad a_{23}=\frac{w_{2}}{2}-B_{4}, \quad a_{24}=\frac{w_{1}}{2}-B_{2}-A_{3}, \\
a_{31}=\frac{w_{1}}{2}+B_{2}+A_{3}, \quad a_{32}=\frac{w_{2}}{2}+B_{4}, \quad a_{34}=\frac{w_{1}}{2}+B_{2}-A_{3}, \\
a_{41}=\frac{w_{1}}{2}+A_{4}, \quad a_{42}=\frac{w_{2}}{2}+A_{2}+B_{3}, \quad a_{43}=\frac{w_{2}}{2}+A_{2}-B_{3},
\end{gathered}
$$


where

$$
\begin{array}{ll}
A_{1}=\frac{1}{2} \sqrt{\frac{15+2 \sqrt{30}}{35}}, \quad A_{2}=A_{1}\left(\frac{1}{6}+\frac{\sqrt{30}}{24}\right), \quad A_{3}=A_{1}\left(\frac{1}{21}+\frac{5 \sqrt{30}}{168}\right), & A_{4}=A_{1}-2 A_{2}, \\
B_{1}=\frac{1}{2} \sqrt{\frac{15-2 \sqrt{30}}{35}}, \quad B_{2}=A_{1}\left(\frac{1}{6}-\frac{\sqrt{30}}{24}\right), \quad B_{3}=B_{1}\left(\frac{1}{21}-\frac{5 \sqrt{30}}{168}\right), \quad B_{4}=B_{1}-2 B_{2},
\end{array}
$$

then the method is of the maximum order $(p=8)$.

For $t_{0}=0$ and $y_{0} \in Y_{0}$ four-stage implicit interval methods are defined by the following set of formulas:

$$
\begin{aligned}
Y_{n}\left(t_{0}\right) & =Y_{n}(0)=Y_{0}, \\
Y_{n}\left(t_{k+1}\right) & =Y_{n}\left(t_{k}\right)+h\left(w_{1} K_{1, k}(h)+w_{2} K_{2, k}(h)+w_{3} K_{3, k}(h)+w_{4} K_{4, k}(h)\right) \\
& +\left(\Psi\left(T_{k}, Y_{n}\left(t_{k}\right)\right)+[-\alpha, \alpha]\right) h^{p+1}, \\
k & =0,1, \ldots, n-1,
\end{aligned}
$$

where $p \leq 8$, and

$$
\begin{aligned}
& K_{i, k}(h)= F\left(T_{k}+c_{i} h,\right. \\
&\left.Y_{n}\left(t_{k}\right)+h\left(a_{i 1} K_{1, k}(h)+a_{i 2} K_{2, k}(h)+a_{i 3} K_{3, k}(h)+a_{i 4} K_{4, k}(h)\right)\right), \\
& i=1,2,3,4, \quad \alpha=M h_{0} .
\end{aligned}
$$

The step-size $h, 0<h \leq h_{0}$, where $h_{0}$ is given, can be found from the formula

$$
h=\frac{\eta_{4}^{*}}{n},
$$

where

$$
\eta_{4}^{*}=\min \left\{\eta_{0}, \eta_{1}, \eta_{2}, \eta_{3}, \eta_{4}\right\}
$$

The numbers $\eta_{i}>0(i=1,2,3,4)$ should fulfilled the conditions

$$
Y_{0}+\eta_{i} c_{i} F\left(\Delta_{i}, \Delta_{y}\right) \subset \Delta_{y}, \quad i=1,2,3,4,
$$

and the number $\eta_{0}$ should be choosen in such a way that

$$
\begin{gathered}
Y_{0}+\eta_{0}\left(w_{1} F\left(\Delta_{t}, \Delta_{y}\right)+w_{2} F\left(\Delta_{t}, \Delta_{y}\right)+w_{3} F\left(\Delta_{t}, \Delta_{y}\right)+w_{4} F\left(\Delta_{t}, \Delta_{y}\right)\right) \\
+\left(\Psi\left(\Delta_{t}, \Delta_{y}\right)+[-\alpha, \alpha]\right) h_{0}^{p} \subset \Delta_{y}
\end{gathered}
$$

for $Y_{0} \subset \Delta_{y}$ and $y_{0} \in Y_{0}$. 
In [6] we proved (Theorem 1 in Sect. 5) that for one- and two-stage implicit interval methods the exact solution of the initial value problem (1) belonged to the intervals obtained by these methods. Since in that theorem there are no restrictions to one- or two-stage methods, the same conclusion we get for the methods (7) - (8) and (13) - (14).

\section{WIDTHS OF INTERVAL SOLUTIONS}

First, let us consider the widths of intervals $\quad K_{i, k}(h)$ given by (8) and (14). From these formulas and properties of the function $\boldsymbol{F}$ (see Sect. 3) it follows that

$$
d\left(K_{i, k}(h)\right) \leq L\left[d\left(T_{k}\right)+d\left(Y_{n}\left(t_{k}\right)\right)\right]+h L \sum_{j=1}^{m}\left|a_{i j}\right| d\left(K_{j, k}(h)\right),
$$

where $m=3, i=1,2,3$ for the method (7) - (8), and $m=4, i=1,2,3,4$ for the method (13) - (14). The inequalities (19) are of the form

$$
\left(1-\alpha_{i i}\right) x_{i}-\sum_{\substack{j=1 \\ j \neq i}}^{m} \alpha_{i j} x_{j} \leq \beta, \quad i=1,2, \ldots, m
$$

For $m=3$ we get the following solution of (20):

$$
\begin{aligned}
& x_{1} \leq \frac{\beta}{W_{3}}\left[\left(1-\alpha_{22}\right)\left(1-\alpha_{33}\right)+\left(1+\alpha_{23}-\alpha_{33}\right) \alpha_{12}+\left(1+\alpha_{32}-\alpha_{22}\right) \alpha_{13}-\alpha_{23} \alpha_{32}\right], \\
& x_{2} \leq \frac{\beta}{W_{3}}\left[\left(1-\alpha_{11}\right)\left(1-\alpha_{33}\right)+\left(1+\alpha_{13}-\alpha_{33}\right) \alpha_{21}+\left(1+\alpha_{31}-\alpha_{11}\right) \alpha_{23}-\alpha_{13} \alpha_{31}\right], \\
& x_{3} \leq \frac{\beta}{W_{3}}\left[\left(1-\alpha_{11}\right)\left(1-\alpha_{22}\right)+\left(1+\alpha_{12}-\alpha_{22}\right) \alpha_{31}+\left(1+\alpha_{21}-\alpha_{11}\right) \alpha_{32}-\alpha_{12} \alpha_{21}\right]
\end{aligned}
$$

where

$$
\begin{aligned}
W_{3} & =\left(1-\alpha_{11}\right)\left(1-\alpha_{22}\right)\left(1-\alpha_{33}\right)-\alpha_{12} \alpha_{23} \alpha_{31}-\alpha_{13} \alpha_{21} \alpha_{32} \\
& -\left(1-\alpha_{11}\right) \alpha_{23} \alpha_{32}-\left(1-\alpha_{22}\right) \alpha_{13} \alpha_{31}-\left(1-\alpha_{33}\right) \alpha_{12} \alpha_{21}
\end{aligned}
$$

and where

$$
\begin{gathered}
1-\alpha_{11}>0, \quad 1-\alpha_{22}>0, \quad 1-\alpha_{33}>0 \\
\left(1-\alpha_{11}\right)\left(1-\alpha_{22}\right)-\alpha_{12} \alpha_{21}>0, \quad\left(1-\alpha_{11}\right)\left(1-\alpha_{33}\right)-\alpha_{13} \alpha_{31}>0 \\
\left(1-\alpha_{22}\right)\left(1-\alpha_{33}\right)-\alpha_{23} \alpha_{32}>0, \quad W_{3}>0 .
\end{gathered}
$$

For $m=4$ the solution of (20) is of the form

$$
\begin{aligned}
x_{1} & \leq \frac{\beta}{W_{4}}\left\{\left(1-\alpha_{22}\right)\left(1-\alpha_{33}\right)\left(1-\alpha_{44}\right)\right. \\
& +\left[1+\left(1+\alpha_{34}-\alpha_{44}\right) \alpha_{23}+\left(1+\alpha_{43}-\alpha_{33}\right) \alpha_{24}-\left(1-\alpha_{44}\right) \alpha_{33}-\alpha_{34} \alpha_{43}-\alpha_{44}\right] \alpha_{12}
\end{aligned}
$$




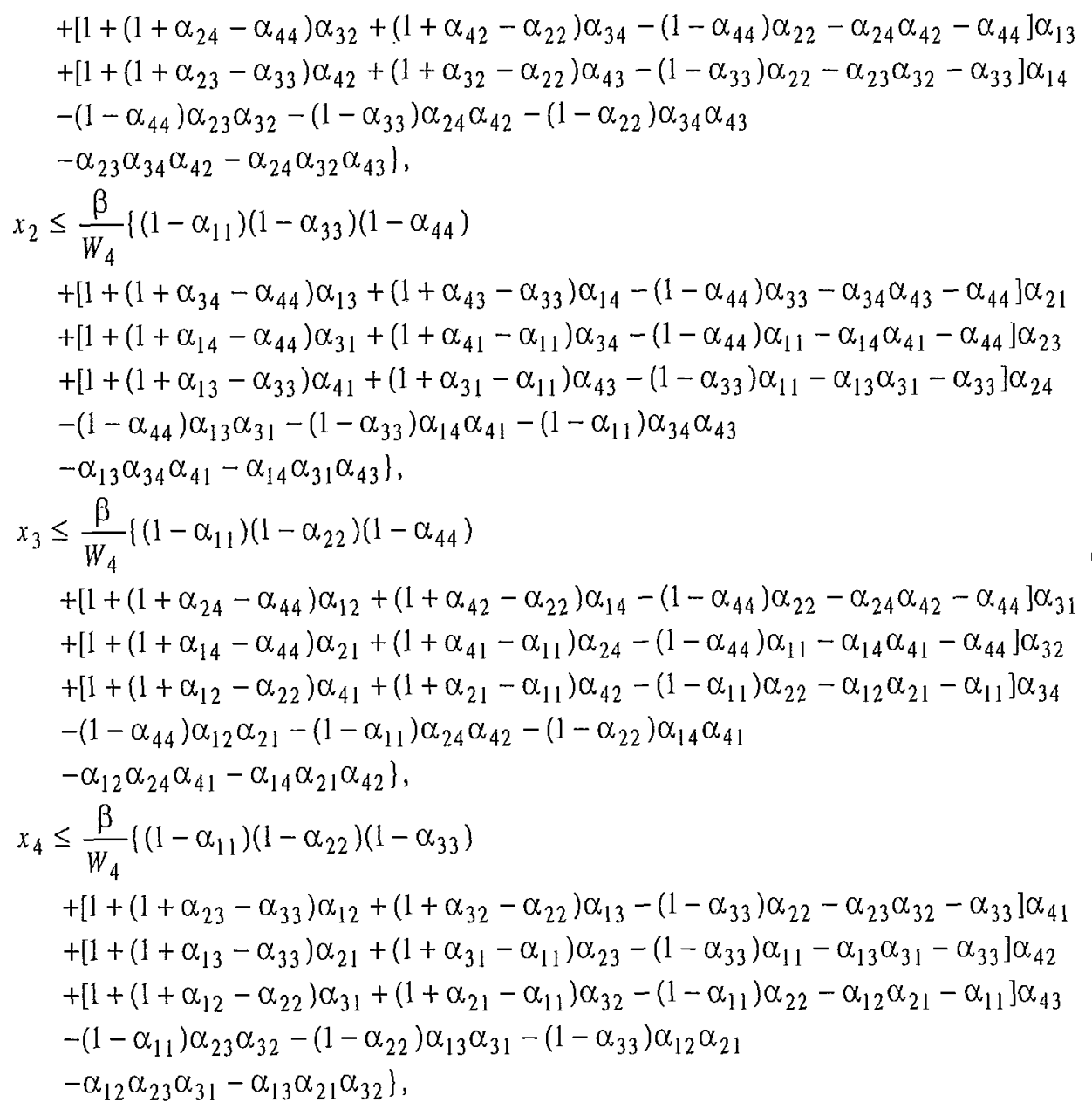

where

$$
\begin{aligned}
W_{4} & =\left(1-\alpha_{11}\right)\left(1-\alpha_{22}\right)\left(1-\alpha_{33}\right)\left(1-\alpha_{44}\right) \\
& -\left(1-\alpha_{44}\right)\left[\left(1-\alpha_{11}\right) \alpha_{23} \alpha_{32}+\left(1-\alpha_{22}\right) \alpha_{13} \alpha_{31}+\left(1-\alpha_{33}\right) \alpha_{12} \alpha_{21}\right] \\
& -\left(1-\alpha_{11}\right)\left(\alpha_{23} \alpha_{34} \alpha_{42}+\alpha_{24} \alpha_{32} \alpha_{43}\right)-\left(1-\alpha_{22}\right)\left(\alpha_{13} \alpha_{34} \alpha_{41}+\alpha_{14} \alpha_{31} \alpha_{43}\right) \\
& -\left(1-\alpha_{33}\right)\left(\alpha_{12} \alpha_{24} \alpha_{41}+\alpha_{14} \alpha_{21} \alpha_{42}\right)-\left(1-\alpha_{44}\right)\left(\alpha_{12} \alpha_{23} \alpha_{31}+\alpha_{13} \alpha_{21} \alpha_{32}\right) \\
& \left.-\alpha_{14} \alpha_{41}\left[\left(1-\alpha_{22}\right)\left(1-\alpha_{33}\right)-\alpha_{23} \alpha_{32}\right]-\alpha_{24} \alpha_{42}\left[\left(1-\alpha_{11}\right)\left(1-\alpha_{33}\right)-\alpha_{13} \alpha_{31}\right)\right] \\
& \left.-\alpha_{34} \alpha_{43}\left[\left(1-\alpha_{11}\right)\left(1-\alpha_{22}\right)-\alpha_{12} \alpha_{21}\right)\right] \\
& -\alpha_{12}\left(\alpha_{23} \alpha_{34} \alpha_{41}+\alpha_{24} \alpha_{31} \alpha_{43}\right)-\alpha_{13}\left(\alpha_{21} \alpha_{34} \alpha_{42}+\alpha_{24} \alpha_{32} \alpha_{41}\right) \\
& -\alpha_{14}\left(\alpha_{21} \alpha_{32} \alpha_{43}+\alpha_{23} \alpha_{31} \alpha_{42}\right),
\end{aligned}
$$


and where

$$
\begin{aligned}
& 1-\alpha_{11}>0, \quad 1-\alpha_{22}>0, \quad 1-\alpha_{33}>0, \quad 1-\alpha_{44}>0 \\
& \left(1-\alpha_{11}\right)\left(1-\alpha_{22}\right)-\alpha_{12} \alpha_{21}>0, \quad\left(1-\alpha_{11}\right)\left(1-\alpha_{33}\right)-\alpha_{13} \alpha_{31}>0 \\
& \left(1-\alpha_{11}\right)\left(1-\alpha_{44}\right)-\alpha_{14} \alpha_{41}>0, \quad\left(1-\alpha_{22}\right)\left(1-\alpha_{33}\right)-\alpha_{23} \alpha_{32}>0 \\
& \left(1-\alpha_{22}\right)\left(1-\alpha_{44}\right)-\alpha_{24} \alpha_{42}>0, \quad\left(1-\alpha_{33}\right)\left(1-\alpha_{44}\right)-\alpha_{34} \alpha_{43}>0 \\
& \left(1-\alpha_{11}\right)\left(1-\alpha_{22}\right)\left(1-\alpha_{33}\right)-\left(1-\alpha_{11}\right) \alpha_{23} \alpha_{32}-\left(1-\alpha_{22}\right) \alpha_{13} \alpha_{31} \\
& \quad-\left(1-\alpha_{33}\right) \alpha_{12} \alpha_{21}-\alpha_{12} \alpha_{23} \alpha_{31}-\alpha_{13} \alpha_{21} \alpha_{32}>0 \\
& \left(1-\alpha_{11}\right)\left(1-\alpha_{22}\right)\left(1-\alpha_{44}\right)-\left(1-\alpha_{11}\right) \alpha_{24} \alpha_{42}-\left(1-\alpha_{22}\right) \alpha_{14} \alpha_{41} \\
& \quad-\left(1-\alpha_{44}\right) \alpha_{12} \alpha_{21}-\alpha_{12} \alpha_{24} \alpha_{41}-\alpha_{14} \alpha_{21} \alpha_{42}>0 \\
& \left(1-\alpha_{11}\right)\left(1-\alpha_{33}\right)\left(1-\alpha_{44}\right)-\left(1-\alpha_{11}\right) \alpha_{34} \alpha_{43}-\left(1-\alpha_{33}\right) \alpha_{14} \alpha_{41} \\
& \quad-\left(1-\alpha_{44}\right) \alpha_{13} \alpha_{31}-\alpha_{13} \alpha_{34} \alpha_{41}-\alpha_{14} \alpha_{31} \alpha_{43}>0 \\
& \left(1-\alpha_{22}\right)\left(1-\alpha_{33}\right)\left(1-\alpha_{44}\right)-\left(1-\alpha_{22}\right) \alpha_{34} \alpha_{43}-\left(1-\alpha_{33}\right) \alpha_{24} \alpha_{42} \\
& \quad-\left(1-\alpha_{44}\right) \alpha_{23} \alpha_{32}-\alpha_{23} \alpha_{34} \alpha_{42}-\alpha_{24} \alpha_{32} \alpha_{43}>0 \\
& W_{4}>0
\end{aligned}
$$

Using the above results we can estimate the widths of interval solutions for implict three- and four-stage methods presented in the previous sections.

Theorem 1. If $Y_{n}\left(t_{k}\right) \quad(k=0,1, \ldots, n)$ are obtained on the basis of the method (7) - (8), then for $h_{0}$ such that

$$
\begin{aligned}
h_{0}<\min \{ & \left\{\frac{1}{L\left|a_{11}\right|}, \frac{1}{L\left|a_{22}\right|}, \frac{1}{L\left|a_{33}\right|}, \frac{1}{L\left(\left|a_{11}\right|+\left|a_{22}\right|\right)+L^{2}\left|a_{12} \| a_{21}\right|},\right. \\
& \frac{1}{L\left(\left|a_{11}\right|+\left|a_{33}\right|\right)+L^{2}\left|a_{13}\right|\left|a_{31}\right|}, \\
& \left.\frac{1}{L\left(\left|a_{22}\right|+\left|a_{33}\right|\right)+L^{2}\left|a_{23} \| a_{32}\right|}, \frac{1}{L \Omega_{1}+L^{2} \Omega_{2}+L^{3} \Omega_{3}}\right\}
\end{aligned}
$$

where

$$
\begin{aligned}
& \Omega_{1}=\left|a_{11}\right|+\left|a_{22}\right|+\left|a_{33}\right| \\
& \Omega_{2}=\left|a_{12}\right|\left|a_{21}\right|+\left|a_{13}\right|\left|a_{31}\right|+\left|a_{23}\right|\left|a_{32}\right| \\
& \Omega_{3}=\left|a_{11}\right|\left|a_{22} \| a_{33}\right|+\left|a_{12}\right|\left|a_{23}\right|\left|a_{31}\right|+\left|a_{13}\right|\left|a_{21}\right|\left|a_{32}\right|
\end{aligned}
$$

we have

$$
d\left(Y_{n}\left(t_{k}\right)\right) \leq Q h^{p}+R d\left(Y_{0}\right)+S \cdot \max _{l=1,2, \ldots, n} d\left(T_{l}\right)
$$

where $Q, R$ and $S$ denote some nonnegative constants, and $p \leq 6$. 
Proof. From (7) we get

$$
\begin{aligned}
d\left(Y_{n}\left(t_{k+1}\right)\right) \leq & d\left(Y_{n}\left(t_{k}\right)\right) \\
& +h\left(\left|w_{1}\right| d\left(K_{1 k}(h)\right)+\left|w_{2}\right| d\left(K_{2 k}(h)\right)+\left|w_{3}\right| d\left(K_{3 k}(h)\right)\right) \\
& +\left[d\left(\Psi\left(\Delta_{t}, \Delta_{y}\right)\right)+2 \alpha\right] h^{p+1},
\end{aligned}
$$

where $p \leq 6, k=0,1, \ldots, n-1$. On the basis of (8) we have

$$
\begin{aligned}
d\left(K_{i, k}(h)\right) \leq & L\left[d\left(T_{k}\right)+d\left(Y_{n}\left(t_{k}\right)\right)\right] \\
& +h L\left(\left|a_{i 1}\right| d\left(K_{1 k}(h)\right)+h L\left|a_{i 2}\right| d\left(K_{2 k}(h)\right)+h L\left|a_{i 3}\right| d\left(K_{3 k}(h)\right)\right), \\
i & =1,2,3 .
\end{aligned}
$$

From (21) it follows that if

$$
\begin{aligned}
& 1-h L\left|a_{i i}\right|>0 \quad i=1,2,3 \\
& \left(1-h L\left|a_{i i}\right|\right)\left(1-h L\left|a_{j j}\right|\right)-h^{2} L^{2}\left|a_{i j}\right|\left|a_{j i}\right|>0, \quad i, j=1,2,3, \quad i \neq j \\
& \left(1-h L\left|a_{11}\right|\right)\left(1-h L\left|a_{22}\right|\right)\left(1-h L\left|a_{33}\right|\right) \\
& -h^{2} L^{2}\left(\left|a_{12}\right|\left|a_{21}\right|+\left|a_{13}\right|\left|a_{31}\right|+\left|a_{23} \| a_{32}\right|\right) \\
& -h^{3} L^{3}\left(\left|a_{12}\left\|a_{23}|| a_{31}|+| a_{13}|| a_{21}\right\| a_{32}\right|\right. \\
& \left.\quad-\left|a_{11}\left\|a_{23}|| a_{32}|-| a_{13}|| a_{22}\right\| a_{31}\right|-\left|a_{12}\left\|a_{21}\right\| a_{33}\right|\right)>0
\end{aligned}
$$

then the solution of inequalities (26) is of the form

$$
d\left(K_{i, k}(h)\right) \leq \frac{U_{i}}{U} L\left[d\left(T_{k}\right)+d\left(Y_{n}\left(t_{k}\right)\right)\right], \quad i=1,2,3,
$$

where

$$
\begin{aligned}
U_{i}= & \left(1-h L\left|a_{j j}\right|\right)\left(1-h L\left|a_{k k}\right|\right)+\left(1+h L\left|a_{j k}\right|-h L\left|a_{k k}\right|\right) h L\left|a_{i j}\right| \\
& +\left(1+h L\left|a_{k j}\right|-h L\left|a_{j j}\right|\right) h L\left|a_{i k}\right|-h^{2} L^{2}\left|a_{j k}\right|\left|a_{k j}\right|, \\
& i, k, j=1,2,3, \quad i \neq k, \quad k \neq j, \quad j \neq i, \\
U= & \left(1-h L\left|a_{11}\right|\right)\left(1-h L\left|a_{22}\right|\right)\left(1-h L\left|a_{33}\right|\right) \\
& -h^{2} L^{2}\left[\left(1-h L\left|a_{11}\right|\right)\left|a_{23}\right|\left|a_{32}\right|+\left(1-h L\left|a_{22}\right|\right)\left|a_{13}\left\|a_{31}\left|+\left(1-h L\left|a_{33}\right|\right)\right| a_{12}\right\| a_{21}\right|\right] \\
& -h^{3} L^{3}\left(\left|a_{12}\left\|a_{23}\right\| a_{31}\right|+\left|a_{13}\right|\left|a_{21}\right|\left|a_{32}\right|\right) .
\end{aligned}
$$

Taking into account the assumption (23), the first six inequalities in (27) are self-evident. The last inequality in (27) is also an implication of this assumption, because from the inequality 


$$
h_{0}<\frac{1}{L \Omega_{1}+L^{2} \Omega_{2}+L^{3} \Omega_{3}}
$$

it follows that for $h \leq h_{0}$ we have

$$
\begin{gathered}
1-h L\left(\left|a_{11}\right|+\left|a_{22}\right|+\left|a_{33}\right|\right)-h L^{2}\left(\left|a_{12}\right|\left|a_{21}\right|+\left|a_{13}\right|\left|a_{31}\right|+\left|a_{23} \| a_{32}\right|\right) \\
-h L^{3}\left(\left|a_{11}\right|\left|a_{22}\left\|a_{33}|+| a_{13}\right\| a_{23}\right|\left|a_{31}\right|+\left|a_{13}\left\|a_{21}\right\| a_{32}\right|\right)>0 .
\end{gathered}
$$

Since $h<1$ (as a consequence of $/_{\mathrm{i} 0}<1$ ), then $h<h$ and $h<h$. Thus, from the last inequality it follows that

$$
\begin{gathered}
1-h L\left(\left|a_{11}\right|+\left|a_{22}\right|+\left|a_{33}\right|\right)-h^{2} L^{2}\left(\left|a_{12} \| a_{21}\right|+\left|a_{13}\right|\left|a_{31}\right|+\left|a_{23}\right|\left|a_{32}\right|\right) \\
-h^{3} L^{3}\left(\left|a_{11}\right|\left|a_{22}\left\|a_{33}|+| a_{13}\right\| a_{23}\left\|a_{31}|+| a_{13}\right\| a_{21} \| a_{32}\right|\right)>0
\end{gathered}
$$

and hence - all the more -

$$
\begin{gathered}
1-h L\left(\left|a_{11}\right|+\left|a_{22}\right|+\left|a_{33}\right|\right)-h^{2} L^{2}\left(\left|a_{12}\right|\left|a_{21}\right|+\left|a_{13}\right|\left|a_{31}\right|+\left|a_{23}\right|\left|a_{32}\right|\right) \\
+h^{2} L^{2}\left(\left|a_{11}\right|\left|a_{22}\right|+\left|a_{11}\right|\left|a_{33}\right|+\left|a_{22} \| a_{33}\right|\right) \\
-h^{3} L^{3}\left(\left|a_{11}\right|\left|a_{22}\left\|a_{33}|+| a_{13}\right\| a_{23}\right|\left|a_{31}\right|+\left|a_{13}\left\|a_{21}\right\| a_{32}\right|\right) \\
+h^{3} L^{3}\left(\left|a_{11}\left\|a_{23}\right\| a_{32}\right|+\left|a_{22}\left\|a_{13}\right\| a_{31}\right|+\left|a_{33}\left\|a_{12}\right\| a_{21}\right|\right)>0 .
\end{gathered}
$$

But the left-hand side of this inequality is equal to the left-hand side of the last inequality in (27).

Taking into account that $h \leq h_{0}$, from (28) we get

$$
\begin{aligned}
d\left(K_{1, k}(h)\right) & \leq \frac{1+h_{0} L\left[\left|a_{12}\right|\left(1+h_{0} L\left|a_{23}\right|\right)+\left|a_{13}\right|\left(1+h_{0} L\left|a_{32}\right|\right)\right]+h_{0}^{2} L^{2}\left|a_{22}\right|\left|a_{33}\right|}{\bar{U}} \\
& \cdot L\left[d\left(T_{k}\right)+d\left(Y_{n}\left(t_{k}\right)\right)\right], \\
d\left(K_{2, k}(h)\right) & \leq \frac{1+h_{0} L\left[\left|a_{21}\right|\left(1+h_{0} L\left|a_{13}\right|\right)+\left|a_{23}\right|\left(1+h_{0} L\left|a_{31}\right|\right)\right]+h_{0}^{2} L^{2}\left|a_{11}\right|\left|a_{33}\right|}{\bar{U}} \\
\cdot L\left[d\left(T_{k}\right)+d\left(Y_{n}\left(t_{k}\right)\right)\right], & \\
d\left(K_{3, k}(h)\right) \leq & \frac{1+h_{0} L\left[\left|a_{31}\right|\left(1+h_{0} L\left|a_{12}\right|\right)+\left|a_{32}\right|\left(1+h_{0} L\left|a_{21}\right|\right)\right]+h_{0}^{2} L^{2}\left|a_{11}\right|\left|a_{22}\right|}{\bar{U}} \\
& \cdot L\left[d\left(T_{k}\right)+d\left(Y_{n}\left(t_{k}\right)\right)\right],
\end{aligned}
$$

where

$$
\begin{aligned}
\bar{U}= & \left(1-h_{0} L\left|a_{11}\right|\right)\left(1-h_{0} L\left|a_{22}\right|\right)\left(1-h_{0} L\left|a_{33}\right|\right) \\
& -h_{0}^{2} L^{2}\left(\left|a_{12} \| a_{21}\right|+\left|a_{13}\right|\left|a_{31}\right|+\left|a_{23}\right|\left|a_{32}\right|\right)-h_{0}^{3} L^{3}\left(\left|a_{12}\left\|a_{23}\right\| a_{31}\right|+\left|a_{13}\left\|a_{21}\right\| a_{32}\right|\right) .
\end{aligned}
$$


Using these estimates, from the inequality (25) we obtain

$$
\begin{gathered}
d\left(Y_{n}\left(t_{k+1}\right)\right) \leq d\left(Y_{n}\left(t_{k}\right)\right)\left(1+v_{3} h L\right)+v_{3} h L d\left(T_{k}\right)+\left[d\left(\Psi\left(\Delta_{t}, \Delta_{y}\right)\right)+2 \alpha\right] h^{p+1}, \\
k=0,1, \ldots, n-1
\end{gathered}
$$

where

$$
\begin{aligned}
v_{3}=\frac{1}{\bar{U}} & \left\{\left|w_{1}\right|\left(1+h_{0} L\left[\left|a_{12}\right|\left(1+h_{0} L\left|a_{23}\right|\right)+\left|a_{13}\right|\left(1+h_{0} L\left|a_{32}\right|\right)\right]+h_{0}^{2} L^{2}\left|a_{22}\right|\left|a_{33}\right|\right)\right. \\
& +\left|w_{2}\right|\left(1+h_{0} L\left[\left|a_{21}\right|\left(1+h_{0} L\left|a_{13}\right|\right)+\left|a_{23}\right|\left(1+h_{0} L\left|a_{31}\right|\right)\right]+h_{0}^{2} L^{2}\left|a_{11} \| a_{33}\right|\right) \\
& \left.+\left|w_{3}\right|\left(1+h_{0} L\left[\left|a_{31}\right|\left(1+h_{0} L\left|a_{12}\right|\right)+\left|a_{32}\right|\left(1+h_{0} L\left|a_{21}\right|\right)\right]+h_{0}^{2} L^{2}\left|a_{11} \| a_{22}\right|\right)\right\} .
\end{aligned}
$$

From (29) it follows that

$$
\begin{aligned}
& d\left(Y_{n}\left(t_{1}\right)\right) \leq d\left(Y_{n}\left(t_{0}\right)\right)\left(1+v_{3} h L\right)+v_{3} h L d\left(T_{0}\right)+\left[d\left(\Psi\left(\Delta_{i}, \Delta_{y}\right)\right)+2 \alpha\right] h^{p+1}, \\
& d\left(Y_{n}\left(t_{2}\right)\right) \leq d\left(Y_{n}\left(t_{1}\right)\right)\left(1+v_{3} h L\right)+v_{3} h L d\left(T_{1}\right)+\left[d\left(\Psi\left(\Delta_{1}, \Delta_{y}\right)\right)+2 \alpha\right] h^{p+1} \\
& \leq\left(d\left(Y_{n}\left(t_{0}\right)\right)\left(1+v_{3} h L\right)+v_{3} h L d\left(T_{0}\right)+\left[d\left(\Psi\left(\Delta_{t}, \Delta_{y}\right)\right)+2 \alpha\right] h^{p+1}\right) \\
& \cdot\left(1+v_{3} h L\right)+v_{3} h L d\left(T_{1}\right)+\left[d\left(\Psi\left(\Delta_{t}, \Delta_{y}\right)\right)+2 \alpha\right] h^{p+1} \\
& =d\left(Y_{n}\left(t_{0}\right)\right)\left(1+v_{3} h L\right)^{2} \\
& \left.+\left(v_{3} h L \cdot \max _{l=0,1} d\left(T_{l}\right)+d\left(\Psi\left(\Delta_{l}, \Delta_{y}\right)\right)+2 \alpha\right] h^{p+1}\right)\left[1+\left(1+v_{3} h L\right)\right], \\
& d\left(Y_{n}\left(t_{3}\right)\right) \leq d\left(Y_{n}\left(t_{2}\right)\right)\left(1+v_{3} h L\right)+v_{3} h L d\left(T_{2}\right)+\left[d\left(\Psi\left(\Delta_{t}, \Delta_{y}\right)\right)+2 \alpha\right] h^{p+1} \\
& \leq\left[d\left(Y_{n}\left(t_{0}\right)\right)\left(1+v_{3} h L\right)^{2}\right. \\
& \left.\left.+\left(v_{3} h L \cdot \max _{l=0,1} d\left(T_{l}\right)+d\left(\Psi\left(\Delta_{l}, \Delta_{y}\right)\right)+2 \alpha\right] h^{p+1}\right)\left(1+\left(1+v_{3} h L\right)\right)\right] \\
& \cdot\left(1+v_{3} h L\right)+v_{3} h L d\left(T_{2}\right)+\left[d\left(\Psi\left(\Delta_{i}, \Delta_{y}\right)\right)+2 \alpha\right] h^{p+1} \\
& \leq d\left(Y_{n}\left(t_{0}\right)\right)\left(1+v_{3} h L\right)^{3} \\
& \left.+\left(v_{3} h L \cdot \max _{l=0,1,2} d\left(T_{l}\right)+d\left(\Psi\left(\Delta_{t}, \Delta_{y}\right)\right)+2 \alpha\right] h^{p+1}\right) \\
& \cdot\left(1+\left(1+v_{3} h L\right)+\left(1+v_{3} h L\right)^{2}\right) \text {, }
\end{aligned}
$$

Thus, for each $k=1,2, \ldots, n$ we have 


$$
\begin{aligned}
d\left(Y_{n}\left(t_{k}\right)\right) \leq & d\left(Y_{n}\left(t_{0}\right)\right)\left(1+v_{3} h L\right)^{k} \\
& +\left(v_{3} h L \cdot \max _{l=0,1, \ldots, k-1} d\left(T_{l}\right)+\left[d\left(\Psi\left(\Delta_{l}, \Delta_{y}\right)\right)+2 \alpha\right] h^{p+1}\right) \sum_{i=0}^{k-1}\left(1+v_{3} h L\right)^{i} .
\end{aligned}
$$

But

$$
\begin{aligned}
\sum_{i=0}^{k-1}\left(1+v_{3} h L\right)^{i} & =\frac{\left(1+v_{3} h L\right)^{k}-1}{v_{3} h L} \\
& \leq \frac{\exp \left(v_{3} k h L\right)-1}{v_{3} h L} \leq \frac{\exp \left(v_{3} h h L\right)-1}{v_{3} h L}=\frac{\exp \left(v_{3} \eta_{3}^{*} L\right)-1}{v_{3} h L} .
\end{aligned}
$$

Hence

$$
d\left(Y_{n}\left(t_{k}\right)\right) \leq R d\left(Y_{n}\left(t_{0}\right)\right)+S \cdot \max _{l=0,1, \ldots, k} d\left(T_{l}\right)+Q h^{p+1}
$$

where

$$
R=\exp \left(\nu_{3} \eta_{3}^{*} L\right), \quad S=R-1, \quad Q=\frac{\exp \left(v_{3} \eta_{3}^{*} L\right)-1}{v_{3} L}\left[d\left(\Psi\left(\Delta_{1}, \Delta_{y}\right)\right)+2 \alpha\right] .
$$

Taking into account that $d\left(T_{0}\right)=0$, from (30) the inequality (24) follows immediately.

Theorem2. If $Y_{n}\left\{t_{k}\right)(k=0,1, \ldots, n)$ are obtained on the basis of the method (13)-(14), then for $h_{0}$ such that

$$
\begin{aligned}
& h_{0}<\min \left\{1, \frac{1}{L\left|a_{11}\right|}, \frac{1}{L\left|a_{22}\right|}, \frac{1}{L\left|a_{33}\right|}, \frac{1}{L\left|a_{44}\right|}\right. \text {, } \\
& \frac{1}{L\left(\left|a_{11}\right|+\left|a_{22}\right|\right)+L^{2}\left|a_{12}\right|\left|a_{21}\right|}, \frac{1}{L\left(\left|a_{11}\right|+\left|a_{33}\right|\right)+L^{2}\left|a_{13}\right|\left|a_{31}\right|} \\
& \frac{1}{L\left(\left|a_{11}\right|+\left|a_{44}\right|\right)+L^{2}\left|a_{14}\right|\left|a_{41}\right|}, \frac{1}{L\left(\left|a_{22}\right|+\left|a_{33}\right|\right)+L^{2}\left|a_{23}\right|\left|a_{32}\right|} \text {, } \\
& \frac{1}{L\left(\left|a_{22}\right|+\left|a_{44}\right|\right)+L^{2}\left|a_{24}\right|\left|a_{42}\right|}, \frac{1}{L\left(\left|a_{33}\right|+\left|a_{44}\right|\right)+L^{2}\left|a_{34} \| a_{43}\right|}, \\
& \frac{1}{L \Theta_{11}+L^{2} \Theta_{12}+L^{3} \Theta_{13}}, \frac{1}{L \Theta_{21}+L^{2} \Theta_{22}+L^{3} \Theta_{23}} \\
& \frac{1}{L \Theta_{31}+L^{2} \Theta_{32}+L^{3} \Theta_{33}}, \frac{1}{L \Theta_{41}+L^{2} \Theta_{42}+L^{3} \Theta_{43}} \text {, } \\
& \left.\frac{1}{L \Xi_{1}+L^{2} \Xi_{2}+L^{3} \Xi_{3}+L^{4} \Xi_{4}}\right\}
\end{aligned}
$$


where

$$
\begin{aligned}
& \Theta_{11}=\left|a_{11}\right|+\left|a_{22}\right|+\left|a_{33}\right| \text {, } \\
& \Theta_{12}=\left|a_{12}\right|\left|a_{21}\right|+\left|a_{13}\right|\left|a_{31}\right|+\left|a_{23}\right|\left|a_{32}\right| \text {, } \\
& \Theta_{13}=\left|a_{11}\right|\left|a_{22}\right|\left|a_{33}\right|+\left|a_{12}\right|\left|a_{23}\right|\left|a_{31}\right|+\left|a_{13}\right|\left|a_{21}\right|\left|a_{32}\right| \text {, } \\
& \Theta_{21}=\left|a_{11}\right|+\left|a_{22}\right|+\left|a_{44}\right| \text {, } \\
& \Theta_{22}=\left|a_{12}\right|\left|a_{21}\right|+\left|a_{14}\right|\left|a_{41}\right|+\left|a_{24}\right|\left|a_{42}\right| \text {, } \\
& \Theta_{23}=\left|a_{11}\right|\left|a_{22}\right|\left|a_{44}\right|+\left|a_{12}\right|\left|a_{24}\right|\left|a_{41}\right|+\left|a_{14}\right|\left|a_{21}\right|\left|a_{42}\right| \text {, } \\
& \Theta_{31}=\left|a_{11}\right|+\left|a_{33}\right|+\left|a_{44}\right| \text {, } \\
& \Theta_{32}=\left|a_{13}\right|\left|a_{31}\right|+\left|a_{14}\right|\left|a_{41}\right|+\left|a_{34}\right|\left|a_{43}\right| \text {, } \\
& \Theta_{33}=\left|a_{11}\right|\left|a_{33}\right|\left|a_{44}\right|+\left|a_{13}\right|\left|a_{34}\right|\left|a_{41}\right|+\left|a_{14}\right|\left|a_{31}\right|\left|a_{43}\right| \text {, } \\
& \Theta_{41}=\left|a_{22}\right|+\left|a_{33}\right|+\left|a_{44}\right| \text {, } \\
& \Theta_{42}=\left|a_{23}\right|\left|a_{32}\right|+\left|a_{24}\right|\left|a_{42}\right|+\left|a_{34}\right|\left|a_{43}\right| \text {, } \\
& \Theta_{43}=\left|a_{22}\right|\left|a_{33}\right|\left|a_{44}\right|+\left|a_{23}\right|\left|a_{34}\right|\left|a_{42}\right|+\left|a_{24}\right|\left|a_{32}\right|\left|a_{43}\right| \text {, } \\
& \Xi_{1}=\left|a_{11}\right|+\left|a_{22}\right|+\left|a_{33}\right|+\left|a_{44}\right| \text {, } \\
& \Xi_{2}=\left|a_{12}\right|\left|a_{21}\right|+\left|a_{13}\right|\left|a_{31}\right|+\left|a_{14}\right|\left|a_{41}\right| \\
& +\left|a_{23}\right|\left|a_{32}\right|+\left|a_{24}\right|\left|a_{42}\right|+\left|a_{34}\right|\left|a_{43}\right| \text {, } \\
& \Xi_{3}=\left|a_{11}\right|\left|a_{22}\right|\left|a_{33}\right|+\left|a_{11}\right|\left|a_{22}\right|\left|a_{44}\right|+\left|a_{11}\right|\left|a_{33}\right|\left|a_{44}\right| \\
& +\left|a_{12}\right|\left|a_{23}\right|\left|a_{31}\right|+\left|a_{12}\right|\left|a_{24}\right|\left|a_{41}\right|+\left|a_{13} \| a_{21}\right|\left|a_{32}\right| \\
& +\left|a_{13}\right|\left|a_{34}\right|\left|a_{41}\right|+\left|a_{14}\right|\left|a_{21}\right|\left|a_{42}\right|+\left|a_{14}\left\|a_{31}\right\| a_{43}\right| \\
& +\left|a_{22}\right|\left|a_{33}\right|\left|a_{44}\right|+\left|a_{23}\right|\left|a_{34}\right|\left|a_{42}\right|+\left|a_{24}\right|\left|a_{32}\right|\left|a_{43}\right| \text {, } \\
& \Xi_{4}=\left|a_{11}\right|\left|a_{22}\right|\left|a_{34}\right|\left|a_{43}\right|+\left|a_{11}\right|\left|a_{23}\right|\left|a_{32} \| a_{44}\right| \\
& +\left|a_{11}\left\|a_{24}|| a_{33}|| a_{42}|+| a_{12}|| a_{21}\right\| a_{33} \| a_{44}\right| \\
& +\left|a_{12}\left\|a_{23}\right\| a_{34}\right|\left|a_{41}\right|+\left|a_{12}\left\|a_{24}\right\| a_{31}\right|\left|a_{43}\right| \\
& +\left|a_{13}\right|\left|a_{21}\right|\left|a_{34}\left\|a_{42}|+| a_{13}|| a_{22}\right\| a_{31}\right|\left|a_{44}\right| \\
& +\left|a_{13}\left\|a_{24}\right\| a_{32}\left\|a_{41}|+| a_{14}\right\| a_{21}\left\|a_{32}\right\| a_{43}\right| \\
& +\left|a_{14}\left\|a_{22}|| a_{33}\right\| a_{41}\right|+\left|a_{14}\left\|a_{23}\right\| a_{31} \| a_{42}\right| \text {, }
\end{aligned}
$$

we have

$$
d\left(Y_{n}\left(t_{k}\right)\right) \leq Q h^{p}+R d\left(Y_{0}\right)+S \cdot \max _{l=1,2, \ldots, n} d\left(T_{l}\right)
$$

where $Q, R$ and $S$ denote some nonnegative constants, and $p \leq 8$. 
Proof. The formulas (13) yield

$$
d\left(Y_{n}\left(t_{k+1}\right)\right) \leq d\left(Y_{n}\left(t_{k}\right)\right)+h \sum_{i=1}^{4}\left|w_{i}\right| d\left(K_{i k}(h)\right)+\left[d\left(\Psi\left(\Delta_{t}, \Delta_{y}\right)\right)+2 \alpha\right] h^{p+1}
$$

where $p \leq 8, k=0,1, \ldots, n-1$. On the basis of (14) we have

$$
\begin{gathered}
d\left(K_{i, k}(h)\right) \leq L\left[d\left(T_{k}\right)+d\left(Y_{n}\left(t_{k}\right)\right)\right]+h L \sum_{j=1}^{4}\left|a_{i j}\right| d\left(K_{j, k}(h)\right), \\
i=1,2,3,4 .
\end{gathered}
$$

From (22) it follows that if

$$
\begin{aligned}
& 1-h L\left|a_{i i}\right|>0, \quad i=1,2,3,4, \\
& \left(1-h L\left|a_{i i}\right|\right)\left(1-h L\left|a_{j j}\right|\right)-h^{2} L^{2}\left|a_{i j} \| a_{j i}\right|>0, \quad i, j=1,2,3,4, \quad i \neq j, \\
& \left(1-h L\left|a_{i i}\right|\right)\left(1-h L\left|a_{j j}\right|\right)\left(1-h L\left|a_{k k}\right|\right) \\
& -h^{2} L^{2}\left[\left(1-h L\left|a_{i i}\right|\right)\left|a_{j k}\right|\left|a_{k j}\right|+\left(1-h L\left|a_{j j}\right|\right)\left|a_{i k} \| a_{k i}\right|\right. \\
& \left.+\left(1-h L\left|a_{k k}\right|\right)\left|a_{i j} \| a_{k j}\right|\right] \\
& -h^{3} L^{3}\left(\left|a_{i j}\left\|a_{j k}\right\| a_{k i}\right|+\left|a_{i k}\left\|a_{j i}\right\| a_{k j}\right|\right)>0 \text {, } \\
& i, j, k=1,2,3,4, \quad i \neq j, \quad j \neq k, \quad k \neq i, \\
& V \equiv\left(1-h L\left|a_{11}\right|\right)\left(1-h L\left|a_{22}\right|\right)\left(1-h L\left|a_{33}\right|\right)\left(1-h L\left|a_{44}\right|\right) \\
& -h^{2} L^{2}\left[\left(1-h L\left|a_{11}\right|\right)\left(1-h L\left|a_{22}\right|\right)\left|a_{34}\right|\left|a_{43}\right|\right. \\
& +\left(1-h L\left|a_{11}\right|\right)\left(1-h L\left|a_{33}\right|\right)\left|a_{24}\right|\left|a_{42}\right| \\
& +\left(1-h L\left|a_{11}\right|\right)\left(1-h L\left|a_{44}\right|\right)\left|a_{23} \| a_{32}\right| \\
& +\left(1-h L\left|a_{22}\right|\right)\left(1-h L\left|a_{33}\right|\right)\left|a_{14}\right|\left|a_{41}\right| \\
& +\left(1-h L\left|a_{22}\right|\right)\left(1-h L\left|a_{44}\right|\right)\left|a_{13} \| a_{31}\right| \\
& \left.+\left(1-h L\left|a_{33}\right|\right)\left(1-h L\left|a_{44}\right|\right)\left|a_{12} \| a_{21}\right|\right] \\
& -h^{3} L^{3}\left[\left(1-h L\left|a_{11}\right|\right)\left(\left|a_{23}\left\|a_{34}|| a_{41}|+| a_{24}\right\| a_{32} \| a_{43}\right|\right)\right. \\
& +\left(1-h L\left|a_{22}\right|\right)\left(\left|a_{13}\left\|a_{34}\right\| a_{41}\right|+\left|a_{14}\left\|a_{31}\right\| a_{43}\right|\right) \\
& +\left(1-h L\left|a_{33}\right|\right)\left(\left|a_{12}\left\|a_{24}\right\| a_{41}\right|+\left|a_{14}\left\|a_{21}\right\| a_{42}\right|\right) \\
& \left.+\left(1-h L\left|a_{44}\right|\right)\left(\left|a_{12}\left\|a_{23}\right\| a_{31}\right|+\left|a_{13}\left\|a_{21}\right\| a_{32}\right|\right)\right]
\end{aligned}
$$




$$
\begin{aligned}
&-h^{4} L^{4} {\left[\left|a_{12}\right|\left(\left|a_{23}\right|\left|a_{34}\left\|a_{41}|+| a_{24}\right\| a_{31}\left\|a_{43}|-| a_{21}\right\| a_{34} \| a_{43}\right|\right)\right.} \\
&+\left|a_{13}\right|\left(\left|a_{21}\left\|a_{34}\right\| a_{42}\right|+\left|a_{24}\left\|a_{32}\right\| a_{41}\right|-\left|a_{24}\left\|a_{31}\right\| a_{42}\right|\right) \\
&\left.+\left|a_{14}\right|\left(\left|a_{21}\left\|a_{32}\right\| a_{43}\right|+\left|a_{23}\left\|a_{31}\right\| a_{42}\right|-\left|a_{23}\left\|a_{32}\right\| a_{41}\right|\right)\right]>0,
\end{aligned}
$$

then

$$
d\left(K_{i, k}(h)\right) \leq \frac{V_{i}}{V} L\left[d\left(T_{k}\right)+d\left(Y_{n}\left(t_{k}\right)\right)\right], \quad i=1,2,3,4,
$$

where

$$
\begin{aligned}
V_{i}= & \left(1-h L\left|a_{j j}\right|\right)\left(1-h L\left|a_{k k}\right|\right)\left(1-h L\left|a_{l l}\right|\right) \\
& +\left[1+h L\left(\left|a_{j k}\right|+\left|a_{j l}\right|\right)-h L\left(\left|a_{k k}\right|+\left|a_{l l}\right|\right)\right] h L\left|a_{i j}\right| \\
& +\left[1+h L\left(\left|a_{k j}\right|+\left|a_{k l}\right|\right)-h L\left(\left|a_{j j}\right|+\left|a_{l l}\right|\right)\right] h L\left|a_{i k}\right| \\
& +\left[1+h L\left(\left|a_{l j}\right|+\left|a_{l k}\right|\right)-h L\left(\left|a_{j j}\right|+\left|a_{k k}\right|\right)\right] h L\left|a_{i l}\right| \\
& -\left(1+h L\left|a_{i j}\right|-h L\left|a_{j j}\right|\right) h^{2} L^{2}\left|a_{k l}\right|\left|a_{k l}\right| \\
& -\left(1+h L\left|a_{i k}\right|-h L\left|a_{k k}\right|\right) h^{2} L^{2}\left|a_{j l}\right|\left|a_{l j}\right| \\
& -\left(1+h L\left|a_{i l}\right|-h L\left|a_{l l}\right|\right) h^{2} L^{2}\left|a_{j k} \| a_{k j}\right| \\
& h^{3} L^{3}\left\{\left|a_{i j}\right|\left[\left|a_{j k}\right|\left(\left|a_{k l}\right|-\left|a_{l l}\right|\right)+\left|a_{j l}\right|\left(\left|a_{l k}\right|-\left|a_{k k}\right|\right)+\left|a_{k k} \| a_{l l}\right|\right]\right. \\
& \quad+\left|a_{i k}\right|\left[\left|a_{k l}\right|\left(\left|a_{l j}\right|-\left|a_{j j}\right|\right)+\left|a_{k j}\right|\left(\left|a_{j l}\right|-\left|a_{l l}\right|\right)+\left|a_{j j}\right|\left|a_{k k}\right|\right] \\
& \left.\quad+\left|a_{i l}\right|\left[\left|a_{l j}\right|\left(\left|a_{j k}\right|-\left|a_{k k}\right|\right)+\left|a_{l k}\right|\left(\left|a_{k j}\right|-\left|a_{j j}\right|\right)+\left|a_{j j}\right|\left|a_{k k}\right|\right]\right\} \\
& -h^{3} L^{3}\left(\left|a_{j k}\right|\left|a_{k l}\right|\left|a_{l j}\right|+\left|a_{j l}\right|\left|a_{k j}\right|\left|a_{l k}\right|\right), \\
& +l=1,2,3,4 i_{i \neq j}, j \neq k, \quad k \neq l, \quad l \neq i,
\end{aligned}
$$

and $V$ is given by (35).

The first fourteen inequalities in (35) are fulfilled from the assumption (31) and because of $h \leq h_{0}$. The last inequality in (35) also follows from (31), because for $h \leq h_{0}$ we have

$$
h<\frac{1}{L \Xi_{1}+L^{2} \Xi_{2}+L^{3} \Xi_{3}+L^{4} \Xi_{4}},
$$




$$
\begin{aligned}
& 1-h L \Xi_{1}-h L^{2} \Xi_{2}-h L^{3} \Xi_{3}-h L^{4} \Xi_{4}>0 \\
& h<h_{0}<1, \quad h^{s}<h(s=2,3,4) . \\
& 1-h L \Xi_{1}-h^{2} L^{2} \Xi_{2}-h^{3} L^{3} \Xi_{3}-h^{4} L^{4} \Xi_{4}>0 \\
& d\left(K_{1, k}(h)\right) \leq \frac{1}{\bar{V}}\left[1+h_{0} L\left(\left|a_{12}\right|+\left|a_{13}\right|+\left|a_{14}\right|\right)\right. \\
& +h_{0}^{2} L^{2}\left(\left|a_{12}\right|\left|a_{23}\right|+\left|a_{12}\right|\left|a_{24}\right|+\left|a_{13}\right|\left|a_{32}\right|\right. \\
& +\left|a_{13}\right|\left|a_{34}\right|+\left|a_{14}\right|\left|a_{42}\right|+\left|a_{14}\right|\left|a_{43}\right| \\
& \left.+\left|a_{22}\right|\left|a_{33}\right|+\left|a_{22}\right|\left|a_{44}\right|+\left|a_{33}\right|\left|a_{44}\right|\right) \\
& +h_{0}^{3} L^{3}\left(\left|a_{12}\left\|a_{23}\right\| a_{34}\right|+\left|a_{12} \| a_{24}\right|\left|a_{43}\right|+\left|a_{12}\right|\left|a_{33}\right|\left|a_{44}\right|\right. \\
& +\left|a_{13}\right|\left|a_{22}\right|\left|a_{44}\right|+\left|a_{13} \| a_{24}\right|\left|a_{32}\right|+\left|a_{13}\right|\left|a_{34}\right|\left|a_{42}\right| \\
& +\left|a_{14}\left\|a_{22}|| a_{33}|+| a_{14}|| a_{23}|| a_{42}|+| a_{14}\right\| a_{32}\right|\left|a_{43}\right| \\
& \left.\left.+\left|a_{22}\left\|a_{34}\right\| a_{43}\right|+\left|a_{23}\left\|a_{32}\right\| a_{44}\right|+\left|a_{24} \| a_{33}\right|\left|a_{42}\right|\right)\right] \\
& \cdot L\left[d\left(T_{k}\right)+d\left(Y_{n}\left(t_{k}\right)\right)\right] \text {, } \\
& d\left(K_{2, k}(h)\right) \leq \frac{1}{\bar{V}}\left[1+h_{0} L\left(\left|a_{21}\right|+\left|a_{23}\right|+\left|a_{24}\right|\right)\right. \\
& +h_{0}^{2} L^{2}\left(\left|a_{11}\right|\left|a_{33}\right|+\left|a_{11}\right|\left|a_{44}\right|+\left|a_{13}\right|\left|a_{21}\right|\right. \\
& +\left|a_{14}\right|\left|a_{21}\right|+\left|a_{23}\right|\left|a_{31}\right|+\left|a_{23}\right|\left|a_{34}\right| \\
& \left.+\left|a_{24}\right|\left|a_{41}\right|+\left|a_{24}\right|\left|a_{43}\right|+\left|a_{33}\right|\left|a_{44}\right|\right) \\
& +h_{0}^{3} L^{3}\left(\left|a_{11}\right|\left|a_{23}\right|\left|a_{44}\right|+\left|a_{11}\right|\left|a_{24}\right|\left|a_{33}\right|+\left|a_{11}\right|\left|a_{34}\right|\left|a_{43}\right|\right. \\
& +\left|a_{13}\right|\left|a_{21}\right|\left|a_{34}\right|+\left|a_{13}\right|\left|a_{24}\right|\left|a_{41}\right|+\left|a_{13}\right|\left|a_{31}\right|\left|a_{44}\right| \\
& +\left|a_{14}\right|\left|a_{21}\right|\left|a_{43}\right|+\left|a_{14}\right|\left|a_{23}\right|\left|a_{31}\right|+\left|a_{14}\right|\left|a_{33}\right|\left|a_{41}\right| \\
& \left.\left.+\left|a_{21}\left\|a_{33}|| a_{44}|+| a_{23}|| a_{34}|| a_{41}|+| a_{24}\right\| a_{31}\right|\left|a_{43}\right|\right)\right] \\
& \cdot L\left[d\left(T_{k}\right)+d\left(Y_{n}\left(t_{k}\right)\right)\right] \\
& d\left(K_{3, k}(h)\right) \leq \frac{1}{\bar{V}}\left[1+h_{0} L\left(\left|a_{31}\right|+\left|a_{32}\right|+\left|a_{34}\right|\right)\right. \\
& +h_{0}^{2} L^{2}\left(\left|a_{11}\right|\left|a_{22}\right|+\left|a_{11}\right|\left|a_{44}\right|+\left|a_{12}\right|\left|a_{31}\right|\right.
\end{aligned}
$$




$$
\begin{aligned}
& +\left|a_{14}\right|\left|a_{31}\right|+\left|a_{21}\right|\left|a_{32}\right|+\left|a_{22}\right|\left|a_{44}\right| \\
& \left.+\left|a_{24}\right|\left|a_{32}\right|+\left|a_{34}\right|\left|a_{41}\right|+\left|a_{34}\right|\left|a_{42}\right|\right) \\
& +h_{0}^{3} L^{3}\left(\left|a_{11}\right|\left|a_{22}\right|\left|a_{34}\right|+\left|a_{11}\right|\left|a_{24}\right|\left|a_{42}\right|+\left|a_{11}\right|\left|a_{32}\right|\left|a_{44}\right|\right. \\
& +\left|a_{12}\right|\left|a_{21}\right|\left|a_{44}\right|+\left|a_{12}\right|\left|a_{24}\right|\left|a_{31}\right|+\left|a_{12}\right|\left|a_{34}\right|\left|a_{41}\right| \\
& +\left|a_{14}\right|\left|a_{21}\right|\left|a_{32}\right|+\left|a_{14}\right|\left|a_{22}\right|\left|a_{41}\right|+\left|a_{14}\right|\left|a_{31}\right|\left|a_{42}\right| \\
& \left.\left.+\left|a_{21}\right|\left|a_{34}\right|\left|a_{42}\right|+\left|a_{22}\right|\left|a_{31}\right|\left|a_{44}\right|+\left|a_{24}\right|\left|a_{32} \| a_{41}\right|\right)\right] \\
& \text { - } L\left[d\left(T_{k}\right)+d\left(Y_{n}\left(t_{k}\right)\right)\right] \text {, } \\
& d\left(K_{4, k}(h)\right) \leq \frac{1}{\bar{V}}\left[1+h_{0} L\left(\left|a_{41}\right|+\left|a_{42}\right|+\left|a_{43}\right|\right)\right. \\
& +h_{0}^{2} L^{2}\left(\left|a_{11}\right|\left|a_{22}\right|+\left|a_{11}\right|\left|a_{33}\right|+\left|a_{12}\right|\left|a_{41}\right|\right. \\
& +\left|a_{13}\right|\left|a_{41}\right|+\left|a_{21}\right|\left|a_{42}\right|+\left|a_{22}\right|\left|a_{33}\right| \\
& \left.+\left|a_{23}\right|\left|a_{42}\right|+\left|a_{31}\right|\left|a_{43}\right|+\left|a_{32}\right|\left|a_{43}\right|\right) \\
& +h_{0}^{3} L^{3}\left(\left|a_{11}\right|\left|a_{22} \| a_{43}\right|+\left|a_{11}\right|\left|a_{23}\right|\left|a_{32}\right|+\left|a_{11}\right|\left|a_{33}\right|\left|a_{42}\right|\right. \\
& +\left|a_{12}\left\|a_{21}\right\| a_{33}\right|+\left|a_{12}\right|\left|a_{23}\right|\left|a_{41}\right|+\left|a_{12}\right|\left|a_{31}\right|\left|a_{43}\right| \\
& +\left|a_{13}\right|\left|a_{21}\right|\left|a_{42}\right|+\left|a_{13}\right|\left|a_{22}\right|\left|a_{31}\right|+\left|a_{13}\right|\left|a_{32}\right|\left|a_{41}\right| \\
& \left.\left.+\left|a_{21}\right|\left|a_{32}\right|\left|a_{43}\right|+\left|a_{22}\right|\left|a_{33}\right|\left|a_{41}\right|+\left|a_{23} \| a_{31}\right|\left|a_{42}\right|\right)\right] \\
& \text { - } L\left[d\left(T_{k}\right)+d\left(Y_{n}\left(t_{k}\right)\right)\right] \text {, }
\end{aligned}
$$

where

$$
\begin{gathered}
\bar{V}=\left(1-h_{0} L\left|a_{11}\right|\right)\left(1-h_{0} L\left|a_{22}\right|\right)\left(1-h_{0} L\left|a_{33}\right|\right)\left(1-h_{0} L\left|a_{44}\right|\right) \\
-h_{0}^{2} L^{2}\left(\left|a_{12}\left\|a_{21}|+| a_{13}\right\| a_{31}\right|+\left|a_{14}\left\|a_{41}|+| a_{23}\right\| a_{32}\right|+\left|a_{24}\left\|a_{42}|+| a_{34}\right\| a_{43}\right|\right) \\
-h_{0}^{3} L^{3}\left(\left|a_{12}\left\|a_{23}\right\| a_{31}\right|+\left|a_{12}\left\|a_{24}\right\| a_{41}\right|+\left|a_{13}\left\|a_{21}\right\| a_{32}\right|+\left|a_{13}\left\|a_{34}\right\| a_{41}\right|\right. \\
\left.\quad+\left|a_{14}\left\|a_{21}\right\| a_{42}\right|+\left|a_{14}\left\|a_{31}\right\| a_{43}\right|+\left|a_{23}\left\|a_{34}\right\| a_{42}\right|+\left|a_{24}\left\|a_{32}\right\| a_{43}\right|\right) \\
-h_{0}^{4} L^{4}\left(\left|a_{12}\left\|a_{23}\right\| a_{34}\left\|a_{41}|+| a_{12}\right\| a_{24}\left\|a_{31}\right\| a_{43}\right|+\left|a_{13}\left\|a_{21}\right\| a_{34} \| a_{42}\right|\right. \\
\left.+\left|a_{13}\left\|a_{24}\right\| a_{32}\left\|a_{41}|+| a_{14}\right\| a_{21}\left\|a_{32}\right\| a_{43}\right|+\left|a_{14}\left\|a_{23}\right\| a_{31} \| a_{42}\right|\right) .
\end{gathered}
$$

Using these estimates, from the inequality (33) we obtain

$$
\begin{gathered}
d\left(Y_{n}\left(t_{k+1}\right)\right) \leq d\left(Y_{n}\left(t_{k}\right)\right)\left(1+v_{4} h L\right)+v_{4} h L d\left(T_{k}\right)+\left[d\left(\Psi\left(\Delta_{l}, \Delta_{y}\right)\right)+2 \alpha\right] h^{p+1}, \\
k=0,1, \ldots, n-1,
\end{gathered}
$$


58

K. Gajda, A. Marciniak, B. Szyszka

where

$$
v_{4}=\frac{1}{\bar{V}} \sum_{i=1}^{4}\left|w_{i}\right|\left(1+\sum_{j=1}^{3} A_{i j} h_{0}^{j} L^{j}\right) \text {, }
$$

and where

$$
\begin{aligned}
& A_{11}=\left|a_{12}\right|+\left|a_{13}\right|+\left|a_{14}\right| \text {, } \\
& A_{12}=\left|a_{12}\right|\left(\left|a_{23}\right|+\left|a_{24}\right|\right)+\left|a_{13}\right|\left(\left|a_{32}\right|+\left|a_{34}\right|\right)+\left|a_{14}\right|\left(\left|a_{42}\right|+\left|a_{43}\right|\right) \\
& +\left|a_{22}\right|\left|a_{33}\right|+\left|a_{22}\right|\left|a_{44}\right|+\left|a_{33}\right|\left|a_{44}\right| \text {. } \\
& A_{13}=\left|a_{12}\right|\left(\left|a_{23}\right|\left|a_{34}\right|+\left|a_{24}\right|\left|a_{43}\right|+\left|a_{33}\right|\left|a_{44}\right|\right) \\
& +\left|a_{13}\right|\left(\left|a_{22}\right|\left|a_{44}\right|+\left|a_{24}\right|\left|a_{32}\right|+\left|a_{34}\right|\left|a_{42}\right|\right) \\
& +\left|a_{14}\right|\left(\left|a_{22}\right|\left|a_{33}\right|+\left|a_{23}\right|\left|a_{42}\right|+\left|a_{32}\right|\left|a_{43}\right|\right) \\
& +\left|a_{22}\right|\left|a_{34}\right|\left|a_{43}\right|+\left|a_{23}\right|\left|a_{32}\right|\left|a_{44}\right|+\left|a_{24}\right|\left|a_{33}\right|\left|a_{42}\right| \text {, } \\
& A_{21}=\left|a_{21}\right|+\left|a_{23}\right|+\left|a_{24}\right| \text {, } \\
& A_{22}=\left|a_{21}\right|\left(\left|a_{13}\right|+\left|a_{14}\right|\right)+\left|a_{23}\right|\left(\left|a_{31}\right|+\left|a_{34}\right|\right)+\left|a_{24}\right|\left(\left|a_{41}\right|+\left|a_{43}\right|\right) \\
& +\left|a_{11}\right|\left|a_{33}\right|+\left|a_{11}\right|\left|a_{44}\right|+\left|a_{33}\right|\left|a_{44}\right| \text {. } \\
& A_{23}=\left|a_{21}\right|\left(\left|a_{13}\right|\left|a_{34}\right|+\left|a_{14}\right|\left|a_{43}\right|+\left|a_{33}\right|\left|a_{44}\right|\right) \\
& +\left|a_{23}\right|\left(\left|a_{11}\right|\left|a_{44}\right|+\left|a_{14}\right|\left|a_{31}\right|+\left|a_{34}\right|\left|a_{41}\right|\right) \\
& +\left|a_{24}\right|\left(\left|a_{11}\right|\left|a_{33}\right|+\left|a_{13}\right|\left|a_{41}\right|+\left|a_{31}\right|\left|a_{43}\right|\right) \\
& +\left|a_{11}\right|\left|a_{34}\right|\left|a_{43}\right|+\left|a_{13}\right|\left|a_{31}\right|\left|a_{44}\right|+\left|a_{14}\right|\left|a_{33}\right|\left|a_{41}\right| \text {. } \\
& A_{31}=\left|a_{31}\right|+\left|a_{33}\right|+\left|a_{34}\right| \text {, } \\
& A_{32}=\left|a_{31}\right|\left(\left|a_{12}\right|+\left|a_{14}\right|\right)+\left|a_{32}\right|\left(\left|a_{21}\right|+\left|a_{24}\right|\right)+\left|a_{34}\right|\left(\left|a_{41}\right|+\left|a_{42}\right|\right) \\
& +\left|a_{11}\right|\left|a_{22}\right|+\left|a_{11}\right|\left|a_{44}\right|+\left|a_{22}\right|\left|a_{44}\right| \text {. } \\
& A_{33}=\left|a_{31}\right|\left(\left|a_{12}\left\|a_{24}|+| a_{14}\right\| a_{42}\right|+\left|a_{22}\right|\left|a_{44}\right|\right) \\
& +\left|a_{32}\right|\left(\left|a_{11}\right|\left|a_{44}\right|+\left|a_{14}\right|\left|a_{21}\right|+\left|a_{24}\right|\left|a_{41}\right|\right) \\
& +\left|a_{34}\right|\left(\left|a_{11}\right|\left|a_{22}\right|+\left|a_{12}\right|\left|a_{41}\right|+\left|a_{21}\right|\left|a_{42}\right|\right) \\
& +\left|a_{11}\right|\left|a_{24}\right|\left|a_{42}\right|+\left|a_{12}\right|\left|a_{21}\right|\left|a_{44}\right|+\left|a_{14}\right|\left|a_{22}\right|\left|a_{41}\right| \text {, } \\
& A_{41}=\left|a_{41}\right|+\left|a_{42}\right|+\left|a_{43}\right| \text {, } \\
& A_{42}=\left|a_{41}\right|\left(\left|a_{12}\right|+\left|a_{13}\right|\right)+\left|a_{42}\right|\left(\left|a_{21}\right|+\left|a_{23}\right|\right)+\left|a_{43}\right|\left(\left|a_{31}\right|+\left|a_{32}\right|\right) \\
& +\left|a_{11}\right|\left|a_{22}\right|+\left|a_{11}\right|\left|a_{33}\right|+\left|a_{22}\right|\left|a_{33}\right| \text {. }
\end{aligned}
$$




$$
\begin{aligned}
A_{43} & =\left|a_{41}\right|\left(\left|a_{12}\right|\left|a_{23}\right|+\left|a_{13}\left\|a_{32}|+| a_{22}\right\| a_{33}\right|\right) \\
& +\left|a_{42}\right|\left(\left|a_{11}\left\|a_{33}|+| a_{13}\right\| a_{21}\right|+\left|a_{23} \| a_{31}\right|\right) \\
& +\left|a_{43}\right|\left(\left|a_{11}\left\|a_{22}|+| a_{12}\right\| a_{31}\right|+\left|a_{21} \| a_{32}\right|\right) \\
& +\left|a_{11}\right|\left|a_{23}\right|\left|a_{32}\right|+\left|a_{12}\left\|a_{21}|| a_{33}|+| a_{13}\right\| a_{22}\right|\left|a_{31}\right|
\end{aligned}
$$

Proceeding further, as in the proof of the previous theorem, we get

$$
\begin{gathered}
d\left(Y_{n}\left(t_{k}\right)\right) \leq R d\left(Y_{n}\left(t_{0}\right)\right)+S \cdot \max _{l=0,1, \ldots, k} d\left(T_{l}\right)+Q h^{p}, \\
R=\exp \left(v_{4} \eta_{4}^{*} L\right), \quad S \doteq R-1, \quad Q=\frac{\exp \left(v_{4} \eta_{4}^{*} L\right)-1}{v_{4} L}\left[d\left(\Psi\left(\Delta_{t}, \Delta_{y}\right)\right)+2 \alpha\right] .
\end{gathered}
$$

Since $d\left(\boldsymbol{T}_{0}\right)=0$, the inequality (32) is an obvious consequence of (37).

\section{References}

[1] Butcher, J. C., The Numerical Analysis of Ordinary Differential Equations. Runge-Kutta and General Linear Methods, J. Wiley \& Sons, Chichester 1987.

[2] Gajda, K., Marciniak, A., Szyszka, B., Inteiyal Methods of Runge-Kutta Type in Floating-Point Internal Arithmetics. Part II [in Polish], Technical Report RB-028/2000, Poznań University of Technology, Institute of Computing Science, Poznań 2000.

[3] Hairer, E., Nørsett, S. P., Wanner, G., Solving Ordinary Differential Equations I. Nonstiff Problems, Springer-Verlag, Berlin, Heidelberg 1987.

[4] Kalmykov, S. A., Šokin, Ju. I., Juldašev, Z. H., Methods of Inteival Analysis [in Russian], Nauka, Novosibirsk 1986.

[5] Krupowicz, A., Numerical Methods of Initial Value Problems of Ordinary Differential Equations [in Polish], PWN, Warsaw 1986.

[6] Marciniak, A., Szyszka, B., One- and Two-Stage Implicit Inteiyal Methods of Runge-Kutta Type, Computational Methods in Science and Technology 5 (1999), 53-65.

[7] Marciniak, A., Interval Methods of Runge-Kutta Type in Floating-Point Inteiyal Arithmetics [in Polish], Technical Report RB-027/99, Poznań University of Technology, Institute of Computing Science, Poznań 1999.

[8] Marciniak, A., Gajda, K., Marlewski, A., Szyszka, B., The Concept of an Object-Oriented System for Solving the Initial Value Problem by Interval Methods of Runge-Kutta Type [in Polish], Pro Dialog 8 (1999), 39-82.

[9] Marciniak, A., Marlewski, A., Inteiyal Representations of Non-Machine Numbers in Object Pascal [in Polish], Pro Dialog 7 (1998), 75-100.

[10] Šokin, Ju. I., Inteiyal Analysis [in Russian], Nauka, Novosibirsk 1981. 